\title{
Large-scale solar wind structures: occurrence rate and geoeffectiveness
}

\author{
Yuri I. Yermolaev, Nadezhda S. Nikolaeva, Irina G. Lodkina and \\ Mikhail Yu. Yermolaev
}

Space Research Institute (IKI), Russian Academy of Sciences, Moscow, Russia

\begin{abstract}
Large-scale phenomena in the solar wind are important elements of heliospheric physics and space weather. On the basis of the OMNI database of interplanetary measurements we identified large-scale structures of solar wind (SW types) for all time intervals during 1976-2000. Our classification includes quasi-steady types: (1) Heliospheric current sheet (HCS), (2) Slow and (3) Fast SW streams, respectively, from closed and open magnetic field structures in the solar corona, and disturbed types: (4) Corotating interaction regions (CIR - compressed regions between slow and fast SW streams), (5) SHEATH (compressed regions ahead of MC/EJECTA) and (6) Magnetic cloud (MC) and (7) EJECTA as well as (8) direct and (9) reverse interplanetary shocks (see catalog on site ftp://ftp.iki.rssi.ru/pub/omni/ and paper [1]). We discuss several preliminary results obtained with our catalog (see more details in http://www.iki.rssi.ru./people/yyermol_ inf.html) including effects on the Space Weather.
\end{abstract}

Keywords: Large-scalr solar wind phenomena, CME, CIR, solar cycle, occurrence rate, geoeffectiveness

PACS: 96.50.Uv, 96.50.Qx, 94.30.Lr

\section{INTRODUCTION}

Investigation of large-scale (with characteristic time scale more than 1 hour) types of streams in the solar wind allows one to study, on the one hand, the largescale phenomena on the Sun and their variations in the solar cycle and, on the other hand, to study a role of large-scale streams in a transfer of energy from the Sun to the Earth and excitation of geomagnetic disturbances $[2,3,4]$. We identified large-scale structures of solar wind (SW types) for every 1-hour point of measurements during 1976-2000 (see paper [1] and site ftp: //ftp.iki.rssi.ru/pub/omni/). The results of our identification are in good agreement with previous results on selection of individual SW types during shorter time intervals (see, for example, papers $[6,5]$ and references therein). In comparison with the previous studies our catalog has following advantages: (1) Simultaneous inclusion in the catalog of various large-scale SW types at sufficiently long intervals of time comparable to the solar cycle, (2) Inclusion of the improved set of SW types, in particular, selection of ICMEs on EJECTA and MCs and, accordingly, SHEATH before EJECTA and before MCs. By means of this catalog the estimations of magnetic flux which is carried away by CMEs from the Sun [7] and efficiency of geomagnetic storm generation by various interplanetary drivers $[8,9,10]$ have been obtained. In this paper we present several results on occurrence rate and geoeffectiveness of SW types obtained on the basis of our catalog of large scale solar wind phenomena (see paper [11] for details).

\section{METHOD OF DATA PROCESSING}

When the types of solar wind streams were classified, we used OMNI database (see http: / / omniweb. gs fc. nasa.gov [12]) for interval 1976-2000 and available world experience in identification of solar wind streams and the standard criteria for following parameters: velocity $V$, density $N$, proton temperature $T$, ratio of thermal to magnetic pressure ( $\beta$-parameter), ratio of measured temperature to temperature calculated on basis of average "velocity-temperature" relation $T / T_{\text {exp }}$, thermal pressure and magnetic field. This method allows us to identify reliably 3 types of quasi-stationary streams of the solar wind (heliospheric current sheet, fast streams from the coronal holes, and slow streams from the coronal streamers), and 5 disturbed types (compression regions in front of incoming fast streams (CIR), and interplanetary manifestations of coronal mass ejections (ICME) that can include magnetic clouds (MC) and EJECTA with the compression region SHEATH preceding them). In contract with EJECTA, MCs have lower temperature, lower ratio of thermal to magnetic pressure ( $\beta$-parameter) and higher, smooth and rotating magnetic field [13]. In addition, we have included into our catalog such events (rare enough) as direct and reverse shock waves, and the rarefaction region RARE.

When we calculated yearly averaged values, we have taken into consideration that the OMNI database contains gaps of the data from 0 to $50 \%$ time of year. This procedure has been made in the assumption that rate of occurrence of the given SW type is similar both in in- 
tervals of data presence and in intervals of data gap. If during chosen year the number of events of selected SW type $N_{e}$ has been registered in interval of data presence $t_{d}$ the normalized number of the given SW type in this year was defined by multiplication of occurrence rate of the given SW type $N_{e} / t_{d}$ to total duration of year $t_{y}$. Error of this estimation decreases with increasing $N_{e}$ and $t_{d}$, and has been estimated as $N_{e}^{-1 / 2}\left(t_{y}-t_{d}\right) / t_{d}$. When we analyzed durations of different SW types, we selected intervals of SW types which have not data gaps at both edges of the intervals.

\section{RESULTS}

Average values and their standard deviations of several plasma and magnetic field parameters (1st rows) and their statistics (2nd rows) for $8 \mathrm{SW}$ types are presented in Table 1 (see paper [1] for details). Both types of compressed regions (CIR and SHEATH) have very close values of parameters while the parameters for 2 types of ICME (EJECTA and MC) are different. Density, thermal and kinetic pressures are significantly higher (but $\beta$ parameter is lower) in MC than in EJECTA.

Normalized numbers per year, average durations, temporal parts in total times of observations and geoeffectiveness (ratio of number of given $\mathrm{SW}$ type leading to magnetic storms with $D_{s t}<-50 \mathrm{nT}$ to total number of this SW type) for various SW types are presented in Table 2 . In contrast with previous Table 1 , Table 2 consists in two sub-types of SHEATH: SHEATH before EJECTA and SHEATH before MC because these sub-types have different numbers per year and durations while MHD parameters and geoeffectivenesses are similar for both subtypes of SHEATH. We believe that this is first estimation of geoeffectiveness of SHEATH. Numbers and geoeffectivenesses of CIR and SHEATH are approximately equal, but they significantly differ for MC and EJECTA. Though geoeffectiveness of EJECTA is lower than MC (with ratio of 1:7), number of EJECTA is significantly higher (with ratio of 12:1) and part of magnetic storms induced by EJECTA is higher than MC. Table 2 presents 25-year averaged parameters (for example, occurrence of 1-hour measurements for various SW type is shown in Figure 1) while time variations of these parameters with solar cycle are presented in Figure 2-5.

Figure 2 shows yearly averaged sunspots (upper panel) and normalized numbers of various SW types. HCS and CIR have maxima in minimum and declining phase of solar cycles, respectively. Probably EJECTA have 2 peaks near solar maxima, at rising and declining phases. Statistics for MC is very low and it is difficult to make a clear conclusion. Durations of various SW types (see Figure 3) have large standard deviations and thier dependence on solar cycle phases can not be found. Temporal

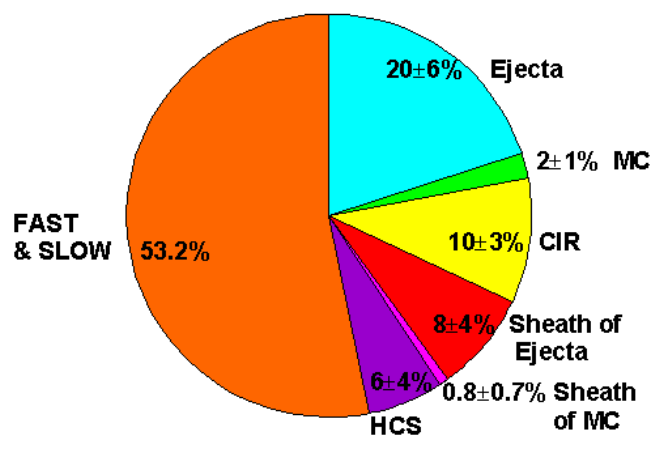

FIGURE 1. Occurance of various SW type measurements during 1976-2000
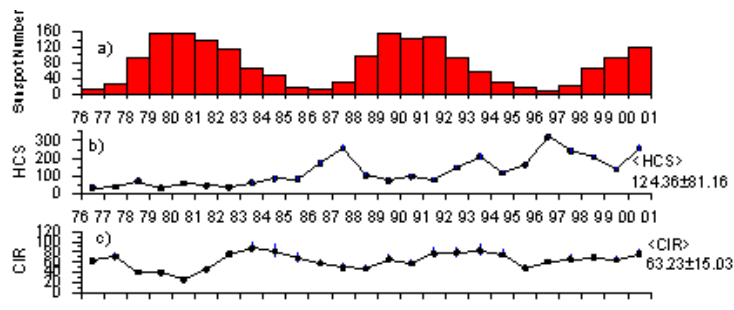

1207677787980818283848586878889909192939495969798990001

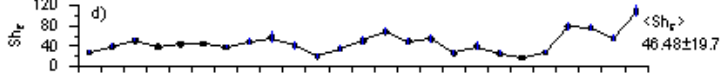

7677787980818283848586878889909192939495969798990001

$\begin{array}{lll}20 & 30\end{array}$

7677787980818283848586878889909192939495969798990001

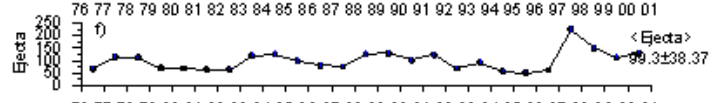

7677787980818283848586878889909192939495969798990001

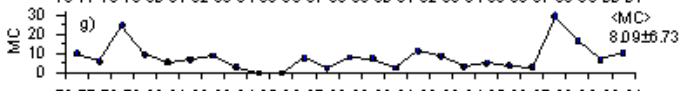

7677787980818283848586878889909192939495969798990001

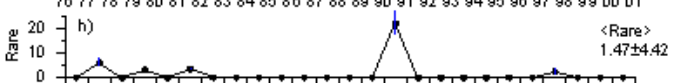

7677787980818283848586878889909192939495969798990001

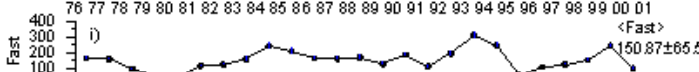

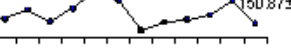

4007677787980818283848586878889909192939495969798990001

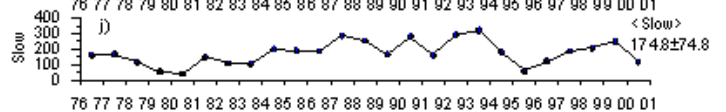

FIGURE 2. Yearly averaged sunspots (upper panel) and normalized numbers of various solar wind types

partions in total times of observations for various SW types (see Figure 4) vary similarly to their normalized numbers (Figure 2).

Figure 5 shows yearly averaged sunspots (upper panel), number of magnetic storms with $D_{s t}<-50 \mathrm{nT}$ (2nd panel) and geoeffectivenesses of various SW types. Geoeffectiveness of CIR is low in minima of solar cycles while geoeffectiveness of EJECTA shows 2 peaks near maxima of solar cycles. 
TABLE 1. Average values and their standard deviations of plasma and magnetic field parameters (1st rows) and their numbers of 1-hour points (2nd rows) for various solar wind types

\begin{tabular}{l|cccccccc}
\hline Parameters & HCS & SLOW & FAST & CIR & EJECTA & MC & SHEATH & RARE \\
\hline$N, \mathrm{~cm}^{-3}$ & $12.1 \pm 6.6$ & $10.8 \pm 7.1$ & $6.6 \pm 5.1$ & $14.1 \pm 9.9$ & $7.8 \pm 5.3$ & $10.1 \pm 8.0$ & $14.3 \pm 10.6$ & $1.7 \pm 1.8$ \\
& 6208 & 84299 & 44543 & 12647 & 27259 & 2225 & 8596 & 139 \\
$V, 10^{2} \mathrm{~km} / \mathrm{s}$ & $3.8 \pm 0.6$ & $3.7 \pm 0.4$ & $5.4 \pm 0.8$ & $4.5 \pm 0.9$ & $4.1 \pm 0.9$ & $4.1 \pm 1.1$ & $4.5 \pm 1.1$ & $5.1 \pm 1.6$ \\
& 6214 & 84805 & 44798 & 12666 & 27310 & 2233 & 8615 & 146 \\
$B, n T$ & $3.9 \pm 2.2$ & $5.9 \pm 2.9$ & $6.4 \pm 3.5$ & $8.7 \pm 4.1$ & $6.4 \pm 2.8$ & $12 \pm 5.2$ & $8.5 \pm 4.5$ & $6.7 \pm 2.2$ \\
& 6322 & 67719 & 36179 & 10493 & 23857 & 2237 & 7286 & 116 \\
$T / T_{\text {exp }}$ & $0.8 \pm 0.9$ & $1.0 \pm 1.4$ & $1.0 \pm 0.7$ & $1.7 \pm 2.0$ & $0.7 \pm 1.3$ & $0.7 \pm 1.5$ & $1.5 \pm 1.2$ & $1.1 \pm 0.9$ \\
$T, 10^{4} \mathrm{~K}$ & 5950 & 75901 & 40026 & 11149 & 25275 & 2016 & 7851 & 124 \\
& $4.1 \pm 4.1$ & $4.4 \pm 4.4$ & $13.1 \pm 11.8$ & $13.8 \pm 13.3$ & $4.2 \pm 5.3$ & $4.5 \pm 6.6$ & $12.9 \pm 17.6$ & $11.1 \pm 10.7$ \\
$N k T, 10^{-2} \mathrm{nPa}$ & 5950 & 75901 & 40026 & 11149 & 25275 & 2016 & 7851 & 124 \\
& $0.6 \pm 1.3$ & $0.6 \pm 1.3$ & $1.3 \pm 2.3$ & $2.2 \pm 2.8$ & $0.4 \pm 1.2$ & $0.7 \pm 2.0$ & $2.2 \pm 3.6$ & $0.3 \pm 0.5$ \\
$m N V^{2}, \mathrm{nPa}$ & 5950 & 75901 & 40026 & 11149 & 25275 & 2016 & 7851 & 124 \\
& $2.9 \pm 1.4$ & $2.4 \pm 1.6$ & $3.2 \pm 2.8$ & $4.4 \pm 2.8$ & $2.1 \pm 1.7$ & $3.3 \pm 3.2$ & $4.9 \pm 4.7$ & $0.8 \pm 0.6$ \\
$\beta, 10^{-1}$ & 6208 & 84299 & 44543 & 12647 & 27259 & 2225 & 8596 & 139 \\
& $9.5 \pm 0.2$ & $5.2 \pm 0.0$ & $6.1 \pm 0.1$ & $6.5 \pm 0.1$ & $3.1 \pm 0.0$ & $1.6 \pm 0.1$ & $6.5 \pm 0.1$ & $2.3 \pm 0.5$ \\
$B_{Z}, \mathrm{nT}$ & 5878 & 59669 & 32244 & 8829 & 20518 & 1725 & 6465 & 100 \\
& $-0.01 \pm 2.3$ & $0.08 \pm 3.1$ & $0.05 \pm 3.4$ & $0.2 \pm 4.4$ & $0.03 \pm 3.3$ & $-0.8 \pm 7.7$ & $0.10 \pm 4.9$ & $0.80 \pm 2.8$ \\
$D_{s t}, \mathrm{nT}$ & 6322 & 67719 & 36179 & 10493 & 23857 & 2237 & 7286 & 116 \\
& $-6.5 \pm 15.0$ & $-10.7 \pm 18.2$ & $-28.7 \pm 25.9$ & $-18.0 \pm 27.2$ & $-21.1 \pm 25.4$ & $-52.1 \pm 45.8$ & $-21.5 \pm 33$ & $-27.0 \pm 22.0$ \\
& 6415 & 85459 & 45017 & 13120 & 29046 & 2571 & 6856 & 147 \\
\hline
\end{tabular}

TABLE 2. Normalized numbers per year, average durations, temporal parts in total times of observations and geoeffectiveness (for magnetic storms with $D_{s t}<-50 \mathrm{nT}$ ) for various solar wind types

\begin{tabular}{l|ccccccccc}
\hline \multirow{2}{*}{ Parameters } & SLOW & \multirow{2}{*}{ FAST } & HCS & \multirow{2}{*}{ CIR } & \multirow{2}{*}{ EJECTA } & MC & \multicolumn{2}{c}{ SHEATH before } & RARE \\
& & & & & & & EJECTA & MC & \\
\hline Number per year & $175 \pm 75$ & $151 \pm 66$ & $124 \pm 81$ & $63 \pm 15$ & $99 \pm 38$ & $8 \pm 7$ & $46 \pm 19$ & $6 \pm 5$ & $1.5 \pm 4.4$ \\
Duration, h & - & - & $5 \pm 2$ & $20 \pm 4$ & $29 \pm 5$ & $25 \pm 12$ & $16 \pm 3$ & $9 \pm 5$ & $4.5 \pm 11$ \\
Time of observation, \% & $31 \pm 7$ & $21 \pm 8$ & $6 \pm 4$ & $10 \pm 3$ & $20 \pm 6$ & $2 \pm 1$ & $8 \pm 4$ & $0.8 \pm 0.7$ & - \\
Geoeffectiveness, \% & - & - & - & 20.2 & 8 & 54.5 & 15.5 & 15.2 & - \\
\hline
\end{tabular}

\section{CONCLUSIONS}

We classified 9 large-scale types of solar wind on the basis of OMNI dataset during 1976-2000 and found.

1. Magnetic clouds and EJECTA have significatly different parameters.

2. Yearly numbers of different structures are $124 \pm$ 81 for HCS, $8 \pm 6$ for MC, $99 \pm 38$ for EJECTA, $46 \pm 19$ for SHEATH before EJECTA, $6 \pm 5$ for SHEATH before MC, and $63 \pm 15$ for CIR.

3. Yearly average durations of phenomena are $5 \pm 2 \mathrm{~h}$ for HCS, $24 \pm 11 \mathrm{~h}$ for MC, $29 \pm 5 \mathrm{~h}$ for EJECTA, $16 \pm 3 \mathrm{~h}$ for SHEATH before EJECTA, $9 \pm 5 \mathrm{~h}$ for SHEATH before MC, and $20 \pm 4 \mathrm{~h}$ for CIR,

4. Solar wind observations consist of $6 \pm 4 \%$ of total time of observations for $\mathrm{HCS}, 2 \pm 1 \%$ for $\mathrm{MC}$, $20 \pm 6 \%$ for EJECTA, $8 \pm 4 \%$ for SHEATH before EJECTA, $0.8 \pm 0.7 \%$ SHEATH before MC, $10 \pm 3 \%$ for CIR.

5. Geoeffectiveness (number of selected SW type resulted in magnetic storms with $D_{s t}<-50 \mathrm{nT}$ di- vided by total number of this SW type) of MC with SHEATH is the largest $(61 \%)$, geoeffectivenesses for CIR and EJECTA with SHEATH are medium (20-21\%) and types of SHEATH and EJECTA without SHEATH have the lowest geoeffectiveness (15 and $8 \%$, respectively).

6. There is a slight indication that number of EJECTA and thier geoeffectiveness have 2 peaks around maxima solar cycles during 1976-2000.

\section{ACKNOWLEDGMENTS}

Paper is supported in part by Physical Department of Russian Academy of Sciences, Program N 15, Presidium of Russian Academy of Sciences, Program N 16, and by RFBR, grants 04-02-16131 and 07-02-00042.

\section{REFERENCES}

1. Yu.I. Yermolaev, N.S. Nikolaeva, I.G. Lodkina, and M.Yu. Yermolaev, Cosmic Res. 47, 81-94 (2009). 


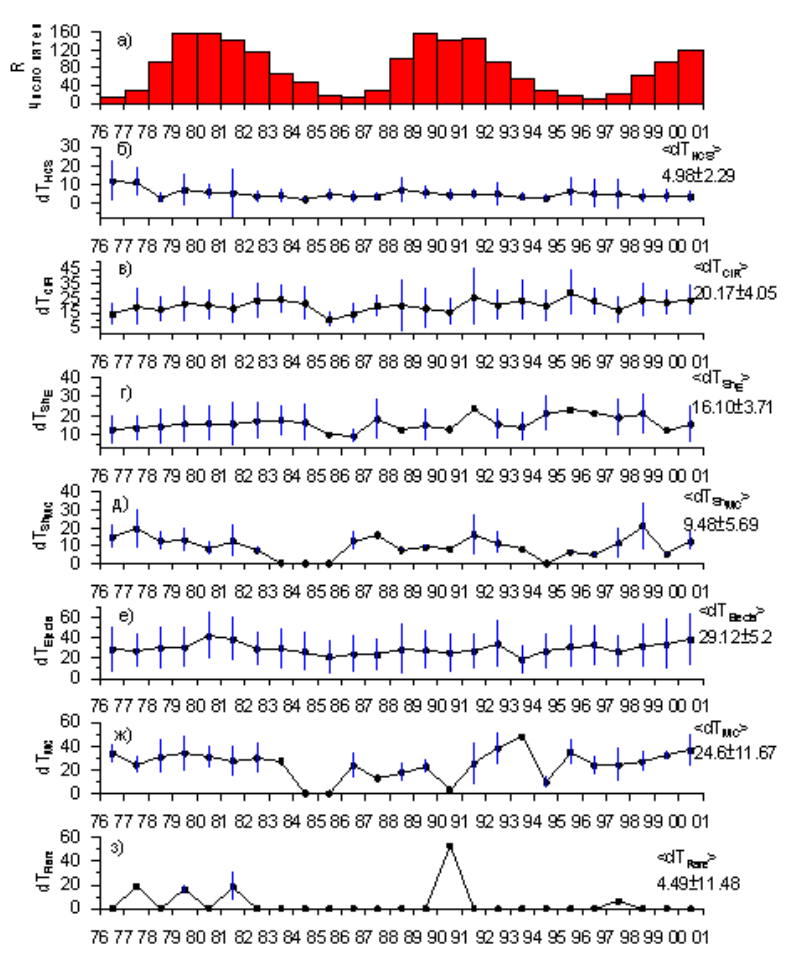

FIGURE 3. Yearly averaged sunspots (upper panel) and durations of various solar wind types

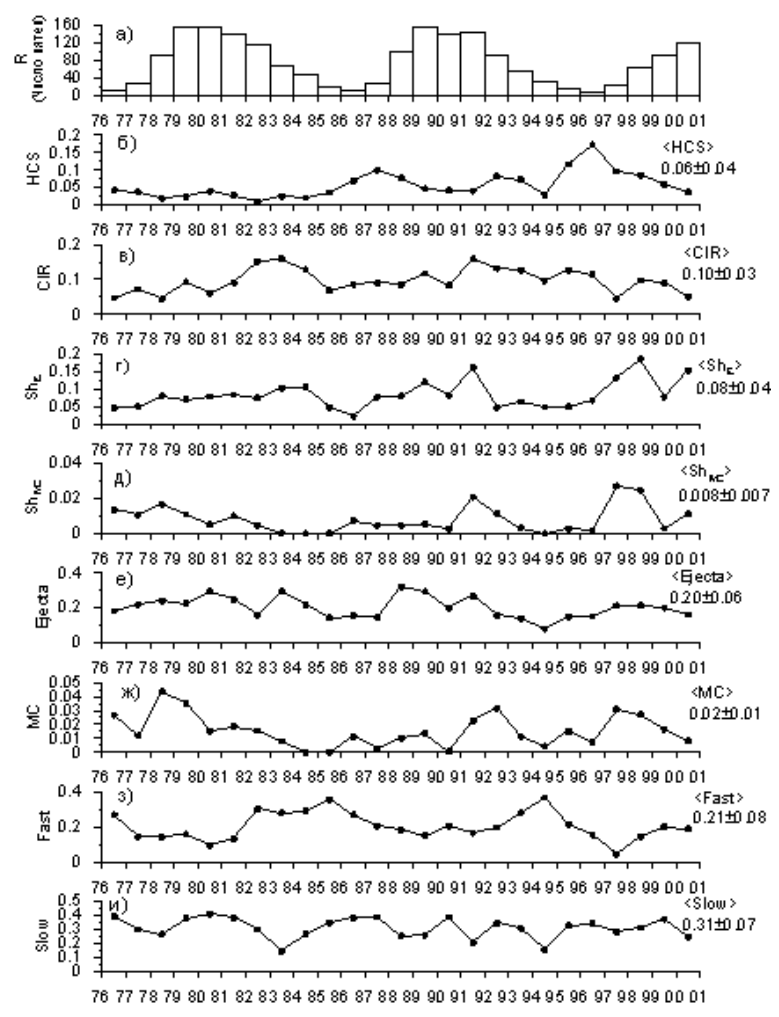

FIGURE 4. Yearly averaged sunspots and temporal parts in total times of observations of various solar wind types
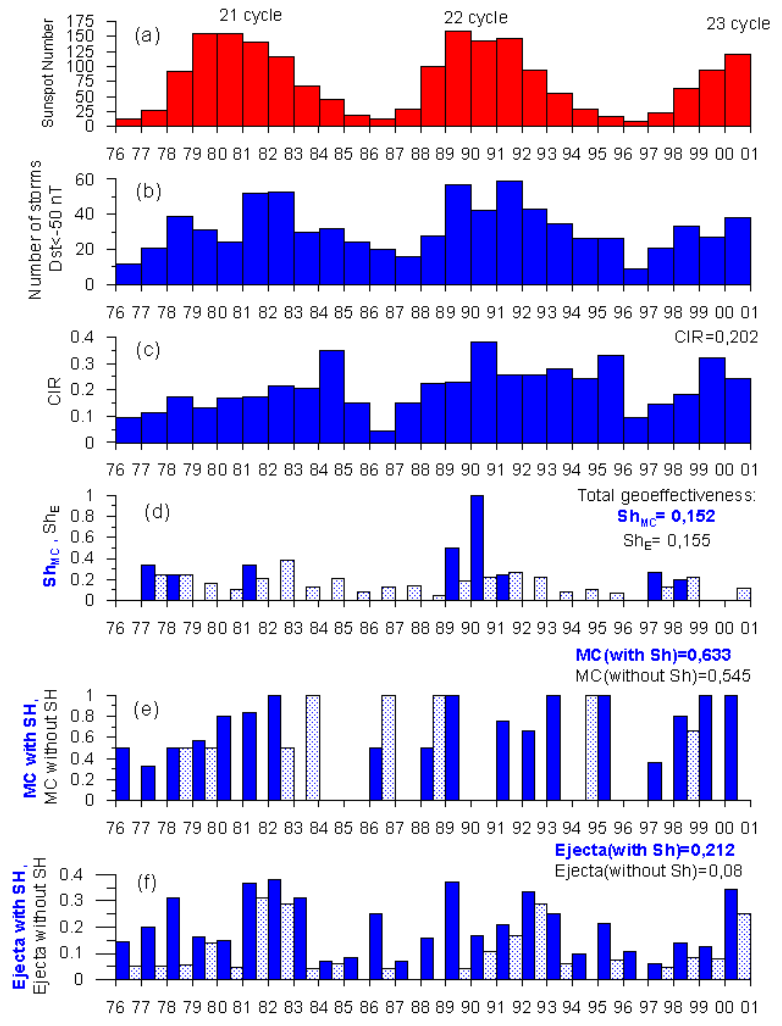

FIGURE 5. Yearly averaged sunspots (1st panel), number of magnetic storms with $D_{s t}<-50 \mathrm{nT}$ (2nd panel) and geoeffectivenesses of various solar wind types

2. B. Fleck, T. H. Zurbuchen, and H. Lacoste (Eds.) Proceedings of the Solar Wind 11 / SOHO 16, "Connecting Sun and Heliosphere" Conference, ESA SP-592 (2005).

3. R. Schwenn. Space Weather: The Solar Perspective, Living Rev. Solar Phys. 3, 2. http://www.livingreviews.org/lrsp2006-2 (2006)

4. T. Pulkkinen, Space Weather: Terrestrial Perspective, Living Rev. Solar Phys. 4, 1. http://www.livingreviews.org/lrsp2007-1 (2007)

5. R.P. Lepping and C.-C. Wu. J. Geophys. Res. 112. A10103. (2007). doi:10.1029/2006JA012140.

6. L.K. Jian, C.T. Russell et al. Solar Phys. 249. P. 85. DOI 10.1007/s11207-008-9161-4. (2008).

7. Yu. I. Yermolaev, I.G. Lodkina, N.S. Nikolaeva, and M.Y. Yermolaev, Solar Phys. 260, (2009, in press).

8. Yu. I. Yermolaev, M.Yu. Yermolaev, N.S. Nikolaeva, and I.G. Lodkina, Bulgarian J. Phys. 34, 128-135 (2007).

9. Yu. I. Yermolaev, M.Yu. Yermolaev, I.G. Lodkina, and N.S. Nikolaeva, Cosmic Res. 45, 3-11 (2007).

10. Yu. I. Yermolaev, M.Yu. Yermolaev, I.G. Lodkina, and N.S. Nikolaeva, Cosmic Res. 45, 489-498 (2007).

11. Yu. I. Yermolaev, N.S. Nikolaeva, I.G. Lodkina, and M.Yu. Yermolaev, Cosmic Res. 48, (2010, in press).

12. J.H. King, and N.E. Papitashvili , J. Geophys. Res. 110 A2. A02209. doi: 10.1029/ 2004JA010804. (2004).

13. L.F. Burlaga, "Magnetic clouds," in Physics of the Inner Heliosphere, edited by R. Schwenn and E. Marsch, Springer-Verlag, New York, 1991. V.2. P.1. 